\title{
PENSAR LA ENSEÑANZA DE LA FILOSOFÍA DESDE LA DIDÁCTICA TEÓRICO-FORMATIVA
}

\author{
Diana Melisa Paredes Oviedo* \\ Universidad de Antioquia \\ Institución Educativa Jesús Rey
}

\section{Resumen}

Los planteamientos didácticos que el pedagogo alemán Wolfang Klafki efectúa desde las ciencias del espíritu, permite repensar la enseñanza de la filosofía de tal manera que los contenidos sean selecciones atendiendo tanto a su pertinencia para la comprensión del mundo del saber, como para la formación intelectual, actitudinal y práctica del sujeto. Es decir, atendiendo a la dialéctica del sujeto y del objeto, o de otro modo, a la relación entre lo sustantivo y lo formal.

Palabras claves: didáctica de la filosofía. Pedagogía y filosofía

\section{Abstract \\ Thinking about philosophy teaching from theoretical-formative didactics}

The didactic approach that the German educator Wolfgang Klafki made from the sciences of spirit, allow us to rethink the teaching of philosophy. In such a way, class contents are pertinent selections that are relevant to the understanding the world of knowledge; as well as to the intellectual, attitudinal and practical formation of the subject; that is to say, in response to the dialectic of subject and object, or in other words, the substantive and the formal.

Key Words: teaching philosophy, pedagogy and philosophy

\section{Generalidades sobre la Didáctica Teórico-Formativa:}

La propuesta didáctica de Klafki se encuentra enmarcada en su concepción de la pedagogía como crítico-constructiva, cuya nota fundamental es que no se debe partir de supuestos teóricos, sino de la realidad educativa, para pensar tanto la enseñanza de saberes como la formación de los sujetos en términos generales. En otras palabras, podríamos afirmar que la Pedagogía debe considerar de manera explícita las problemáticas sociales, que serán abordadas metodológicamente desde la empiria, la crítica ideológica y la hermenéutica, con miras a adelantar un proceso de análisis que permita descubrir las formas de falsa conciencia que influyen de manera subrepticia sobre los individuos y que determinan las maneras de enseñar y aprender.

\footnotetext{
* Licenciada en Filosofía de la Universidad de Antioquia. Estudiante de Maestría en Filosofía en el Instituto de Filosofía, Universidad de Antioquia y Maestría en Educación en la Facultad de Educación, Universidad de Antioquia. Investigadora del Grupo Formaph sobre Formación y Antropología Pedagógica e Histórica clasificado en Categoría A Colciencias; Investigadora del Grupo Filosofía y Pedagogía, reconocido por Colciencias, e Investigadora del Grupo de Investigación de Filosofía Política, Categoría A Colciencias. calebita33@yahoo.com; calebita33@gmail.com.
} 
Lo anterior parece desalentador, pero Klafki da un paso más allá y nos muestra que la Pedagogía que él nos propone aparece como ciencia desde la praxis y para la praxis y, en función de lo anterior, debe tratar de cambiar de manera constructiva la realidad educativa y la enseñanza. Se deben explorar las mejores alternativas para la escuela; pero para su concreción, atendiendo tal objetivo, Klafki señala que se hace necesaria la selección de condiciones para hacerlas posibles, aunque tal proceso debe ser realizado a través de un análisis científico basado en investigaciones empíricas. En otros términos podríamos señalar que en la propuesta pedagógica de Klafki se articulan la crítica ideológica, la reflexión sobre las alternativas posibles, la empiria, la hermenéutica, su concepto de formación general, con su idea de una escuela altamente democratizada y de una enseñanza pensada a partir de una didáctica teórico-formativa.

Este tipo de didáctica debe estar abierta a los asuntos políticos de nuestro tiempo, los cuales se nos muestran en calidad de problemas clave de la contemporaneidad, lo que exige tenerlos en cuenta en una reflexión pedagógica emancipatoria y dentro de una teoría de la formación como las propuestas por Klafki. Para el autor mencionado, la formación general es un principio articulador de la enseñanza y juega un papel importante en esta y, en general, en la educación:

si se quiere impedir [...] que un cúmulo de decisiones y proyectos didácticos se disperse en multitud de actos divergentes [...], se necesita un concepto que designe a esa estructura dinámica global a la que el joven individuo humano debe procurar llegar gradualmente mediante apropiación y vivificación personal de determinadas motivaciones, convicciones, experiencias, habilidades, y que luego habrá de desarrollar y confirmar productivamente en un proceso de integración de nuevas y nuevas experiencias [...]. Formación significa -en la perspectiva de la didácticanada más y nada menos que la quintaesencia de este [...] encargo global [...]; y toda teoría de la didáctica que renuncia a este concepto de formación presupone siempre implícitamente algo equivalente ${ }^{1}$.

Es plausible señalar que en Klafki se hacen presentes las ideas educativas de Comenio $\mathrm{y}$, de manera esencial, lo relacionado con su concepción de "enseñarle a todos todo y de una manera integral o total", así como otras consignas de la Ilustración. Podríamos afirmar que la formación en Klafki es para todos, es decir: se requiere igualdad de oportunidades para todos los individuos; es total e íntegra, en la medida en que su meta debe ser el desarrollo de competencias e intereses variados. En consecuencia, salta a la vista que el pedagogo alemán recupera el concepto de Formación General para la pedagogía y la didáctica, pero le agrega además un tono político.

De acuerdo con lo propuesto por este pensador, lo formativo se abre además a los problemas actuales de las sociedades y no sólo a un canon establecido o a lo clásico, sino también aquello que interese a los educandos: "la enseñanza debe transmitir el pasado y el presente y prever el futuro"2; lo anterior implica que el concepto de aprendizaje sea modificado y debe abarcar aspectos cognitivos, emocionales y sociales, no orientarse por simples resultados o productos, sino enfocarse en los procesos.

\footnotetext{
${ }^{1}$ Klafki, citado por Blankertz, Herwig: "Didáctica”. En: Speck, J. y WEHLE, G y otros: Conceptos fundamentales de pedagogía. Barcelona: Herder, 1981, p. 170.

${ }^{2}$ Runge Peña, Andrés Klaus: Ensayos sobre pedagogía alemana. Bogotá: Universidad Pedagógica Nacional- Museo Pedagógico Colombiano, 2008, p. 167.
} 
Los problemas contemporáneos se deben tratar en la enseñanza de cualquier disciplina, en este caso de la filosofía, a partir de las preguntas por la paz, la destrucción del ecosistema; la pregunta por la desigualdad social, la interculturalidad, las posibilidades y problemas que nos presentan los nuevos medios y las relaciones entre los sujetos en términos de yo-tú. Podría decirse que el concepto de formación de Klafki se encuentra relacionado con la Ilustración europea, que se preocupaba por liberar al hombre de las coacciones sociales y conducirlo a ser un individuo autónomo y responsable.

Según Klafki, la tarea del didacta consiste en trabajar sobre los contenidos y explotar su aspecto formativo, en virtud de ello establece una diferencia clave dentro de su teoría didáctica entre "Inhalt" y "Gehalt"; el primero se debe entender como contenido y el segundo como contenido formativo; en virtud de ello, el autor propone trabajar el Gehalt del Inhalt o, en otros términos, lo formativo que se encuentra en los contenidos. En otras palabras, tanto el didacta de la Filosofía como el educador que se ocupe también de la enseñanza de la Filosofía deberán buscar y explotar los contenidos formativos en las propuestas filosóficas o lo verdaderamente importante, de peso y trascendencia que hay en un contenido filosófico e integrarlo a la enseñanza de esta disciplina; cabe anotar que el contenido aparece como tal en la medida en que transmite un sentido que él mismo guarda y mantiene de una forma particular.

El sentido de los contenidos se puede hacer visible pero se requiere que el didacta se ubique en el contexto de sentido que engloba al contenido, eso significa mirarlos desde su perspectiva pedagógico-formativa. El camino para lograr tal acercamiento es la hermenéutica, como forma de aproximación a la verdad, pero a la vez es la búsqueda misma de ésta. Se trata, entonces, de buscar lo significativo, es decir: lo importante desde el punto de vista pedagógico y formativo que se encuentra en el contenido, lo significativo es entonces "cuando un contenido (Inhalt) tiene contenido formativo (Gehalt)". Klafki nos propone una concepción filosófica y pedagógica de la formación que resulta determinante para el análisis y planeación de la enseñanza. La didáctica aparece para Klafki como resultado de un proceso hermenéutico que presupone la comprensión y la capacidad de transmitir: "El maestro se debe apropiar entonces de un contenido, de tal manera que reconozca en él lo significativo y pueda transmitirlo con el fin de que, a partir de esa mediación, tenga lugar en el proceso formativo"”3.

Pero podemos preguntarnos ¿en qué radica el cambio que propone Klafki en cuanto a la formación de los sujetos? Este autor parte del concepto de formación categorial ${ }^{4}$ para diferenciarse de las teorías formales de la formación, centradas en el sujeto y sus competencias, y las teorías materiales de la formación, que se centran en los contenidos, otorgándoles un objetivismo muy alto, en la medida en que absolutizan los contenidos culturales y los desliga de su historicidad, dotándolos de una validez que hace imposible la aparición de cualquier criterio pedagógico de elección. Las teorías formales de la formación no tienen claridad frente a los contenidos, no dan criterios frente a ellos, ni señalan cuáles serían aquellos que ayuden a la formación de las fuerzas y potencias de los individuos; se pasa por alto también en esta perspectiva que las fuerzas y energías de

\footnotetext{
${ }^{3}$ Ibídem, p. 170.

${ }^{4}$ Para la formulación de una teoría categorial de la formación, Klafki se apoya en la propuesta de Theodor Litt, quien recurre a los tres movimientos del sistema hegeliano para plantear sus teorías sobre la formación. La propuesta categorial surge para subsanar las deficiencias del modelo funcional, fundamentando dialécticamente la relación que se da entre el Yo y el No-Yo, el Hombre y el mundo, el niño y la materia.
} 
los sujetos tienen que ser ejercitadas en y con el mundo. Es decir, sólo en relación con un determinado contenido cobra sentido el concepto de fuerza, energía, espíritu ${ }^{5}$.

Desde la perspectiva de la formación categorial, defendida por este autor, el hombre se forma cuando en la enseñanza se apropia categorialmente de la realidad cultural para abrirse inmediatamente a ella. En la idea de formación de Klafki nos abrimos a un intercambio entre la objetividad del mundo y la subjetividad del individuo, intentando de esta manera superar la antinomia sujeto-objeto y devolviéndosele al contenido la importancia que se merece. El punto es concebir lo otro, lo externo, como prerrequisito dialéctico del yo y centrar el interés de la relación como dominio del mundo y génesis de la persona; en otros términos: "con la idea de formación categorial se busca mediar entre las exigencias del mundo objetivo y el derecho del sujeto de ser él mismo"6. Según Klafki, el hombre es capaz de darle su forma al mundo; la individualidad es capaz de condicionar una perspectiva específica frente al mundo que debe actuar de manera formativa; pero, por el otro lado, nos encontramos con la materia del mundo a partir de la cual el individuo es capaz de elaborar sus contenidos formativos, en otras palabras, podría decirse que el individuo sólo puede formarse cuando se ve a sí mismo frente al mundo; al respecto Klafki nos dice: "Denominamos formación a ese fenómeno en el que -por una vivencia propia o mediante la comprensión de otras personasinteriorizamos inmediatamente la unidad de un momento objetivo (material) y subjetivo (formal)",

La formación categorial se concreta en un doble sentido: la realidad se le abre al hombre categorialmente, y el hombre está abierto para una realidad: conocemos y disponemos de las fuentes culturales del pasado, pero desplegamos de modo armónico y óptimo las capacidades y habilidades propias de los individuos. Formación puede entenderse como el estar abierto a una realidad espiritual y objetiva para el hombre (aspecto sustantivo $u$ objetivo); ese hombre tiene la condición de estar abierto a esa realidad (aspecto subjetivo o formal) tanto desde una perspectiva funcional como metódica. En la formación el hombre se autorrealiza, comprendiéndose a sí mismo; en y mediante el mundo, uno llega a ser uno mismo en el otro, por medio de un proceso dialéctico de reconciliación: en la idea de formación de Klafki se sintetizan el sujeto y el objeto, el alma y el mundo.

En la enseñanza de la Filosofía, así como de cualquier disciplina, desde la perspectiva de Klafki, hay que establecer contenidos formativos que, habida cuenta de las respectivas edades, forman la conciencia y vienen a ser formativos del pensamiento y de la actitud de los sujetos; con base en lo anterior es posible plantear la siguiente pregunta: ¿De qué manera los educandos pueden, en la enseñanza, apropiarse de los contenidos culturales elementales que posibiliten el proceso formativo mencionado? Según Klafki, cuando le mostramos al estudiante los contenidos concretos se le permite reconocer el general implícito en ello, lográndose así la llamada interacción con el mundo: la realidad se abre al estudiante y el estudiante se abre a la realidad; a partir de tal proceso el estudiante adquiere categorías o conceptos fundamentales que sirven como presupuestos y principios de conocimiento y de comprensión del mundo, que puede utilizar y aplicar de manera autónoma; al respecto, el autor nos dice que "el devenir visible de los 'contenidos generales', de los principios categoriales en el

\footnotetext{
${ }^{5}$ Cf. Klafki, Wolfgang: Studien zur Bildungstheorie und Didaktik. Weinheim; Basel: Beltz, 1975, pp. 36 y ss.

${ }^{6}$ Runge, op. cit., p. 172.

${ }^{7}$ Klafki, op. cit.
} 
'material' paradigmático, es decir, de lado de la realidad, no es otra cosa que la consecución de 'categorías' del lado del sujeto" "; los contenidos que forman en ese sentido y transmiten categorías como las planteadas, el autor los denomina "elementaria".

\section{Características del Análisis Didáctico}

En la situación dialéctico-formativa del sujeto y el mundo, propia de la perspectiva categorial, Klafki reconoce tres dimensiones: la elemental con el ejemplo se hace visible y reconocible un principio válido que es aplicable a muchas otros asuntos; la fundamental, referida a la mediación de apreciaciones y transmisión de experiencias fundamentales al educando y que le permiten, mediante la experiencia, reconocer las cosas y que deje una impresión profunda. Lo ejemplar son contenidos que no sólo existen para sí, sino que se abren o permiten abrirse a otros contenidos, nos permiten hacer ese movimiento, le dan una estructura al campo de aprendizaje y se busca un orden en la cabeza de los educandos, en otras palabras, podríamos señalar psicológicamente la presencia de conceptos básicos que nos permitan la adquisición de otros conceptos más elevados que nos llevan a niveles de complejidad diferentes.

Si tomamos un contenido de enseñanza en filosofía y lo observamos a la luz de esas tres dimensiones pedagógico-didácticas, se justifica el análisis de la estructura del contenido. En términos de la formación, en la perspectiva de Klafki, los aspectos pedagógicos no se encuentran supeditados a las dinámicas mismas de los contenidos (objetivo), sino que los parámetros didáctico-formativos son los que guían tales cuestionamientos. La planeación didáctica va primero, orienta el contenido y el método de transmisión. Los principios didácticos permiten delimitar los contenidos de la enseñanza al punto que nos hacen posible señalar qué contenidos deben ser enseñados y cuáles no.

La reducción didáctica opera gracias al análisis didáctico y no queda en manos de las dinámicas de los contenidos en sí mismos. Por un lado, entonces, tenemos a las ciencias o disciplinas que ofrecen información y competencias con relación a los contenidos, en este caso la Filosofía, y, por otra parte, tenemos a la didáctica, que crea las condiciones para que ese objeto o contenido se transforme en contenido formativo, en sentido de una

\footnotetext{
${ }^{8}$ Ibídem, p. 43.

${ }^{9}$ Los elementaria son contenidos básicos a partir de los cuales el sujeto categoriza el mundo; el autor propone siete formas de lo elemental:
}

1- Lo fundamental, que se puede experimentar sólo en términos de vivencia. Las experiencias y vivencias fundamentales solo se pueden desplegar, no definir; ocurren en situaciones específicas no necesariamente planeadas, teniendo como sustrato el hecho de que aparecen estados de ánimo difíciles de ser conceptualizados.

2- Lo ejemplar, que se entiende como aquello según lo cual algo general se experimenta con algo particular, en otros términos: lo general se abstrae de lo particular.

3- Lo típico, a partir del cual lo general se experimenta en lo particular y se nos revela como tipo.

4- Lo clásico, donde lo general se experimenta a partir de un valor o valoración.

5- Lo representativo, según lo cual lo general se experimenta como actualización, como presentificación

6- Las formas finales simples en donde lo general se aprende en un hacer o realización de lo particular, sobresaliendo aquí el carácter práctico.

7- Las formas estéticas simples, en donde lo general se vuelve visible mediante lo único. Podemos experimentar lo general en algo concreto que se nos revela como experiencia única. 
formación categorial que incluya lo material y lo formal, el sujeto y el mundo. Todo lo anterior nos muestra cómo la propuesta didáctica de Klafki (teórico-formativa) reinterpretó de un modo didáctico el concepto neohumanista de formación, logrando que aparezca como útil para la enseñanza. El análisis didáctico se entiende como la interpretación y estructuración didácticas con miras a una planeación de la enseñanza.

Con base en lo anterior, puedo decir que Klafki pone en el centro de su propuesta formativa los contenidos de enseñanza y no los métodos, es decir: los contenidos educativos se deben analizar a partir de sus contenidos formativos, ese es el análisis didáctico, que se puede plantear como cinco preguntas básicas a partir de las cuales el maestro cuestiona de manera crítica los contenidos. Todo educador que pretenda trabajar con contenidos educativos o de enseñanza en filosofía, desde una perspectiva formativa, debe analizarlos de acuerdo con estas cinco dimensiones, y si soportan tal análisis se legitima la enseñanza de estos desde una perspectiva teórico-formativa.

Las preguntas didácticas para formular son:

1) El significado ejemplar del contenido: ¿Qué contexto general de sentido o del mundo soporta o abarca ese contenido? ¿Qué fenómeno fundamental, principio básico, criterio, problema, método, técnica, actitud se puede comprender de una manera ejemplar a partir de la relación con ese contenido? ¿De qué debería ser ejemplar, representativo, típico de ese tema? ¿En dónde se puede hacer fructífero posteriormente el aporte de ese tema en tanto totalidad o en elementos particulares (apreciaciones, representaciones, conceptos valorativos, métodos de trabajo, técnicas)?

2) Importancia para el presente: ¿Qué importancia tiene el respectivo contenido en la vida espiritual del niño o del educando? ¿Qué importancia debería tener tal contenido desde el punto de vista pedagógico?

3) Importancia para el futuro: ¿En qué consiste la importancia del tema para el futuro del niño o del educando? Se debe exigir que la enseñanza explique las cosas y fortalezca a niños y a jóvenes para que logren actuar de forma adecuada bajo las características de un futuro incierto.

4) Estructura del contenido: ¿Cuál es la estructura del contenido, según las preguntas pedagógicas de la primera, segunda y tercera dimensiones? ¿Cuáles son los momentos particulares del contenido y en qué conexión se encuentran esos momentos particulares? ¿En una conexión lógica, fáctica? ¿Tiene niveles el contenido? ¿Tiene diferentes niveles de sentido y de importancia? ¿Pueden ser comprendidos esos niveles de manera independiente entre sí? ¿En qué contexto mayor se encuentra ese contenido? ¿Qué propiedades del contenido podrían hacerle difícil al educando su apropiación? ¿Qué se tiene que mantener como saber mínimo para que el contenido pueda ser tenido como un contenido espiritual 'vivo' y efectivo?

5) Asequibilidad del contenido: ¿Cuáles son los sucesos, situaciones, intentos a partir de los cuales los niños de esa clase, de ese nivel pueden, de una manera interesada, cuestionar, comprender, contrariar, intuir, acceder a la estructura del respectivo contenido? ¿Cuáles son las situaciones, observaciones, intuiciones, intentos, modelos apropiados para que los educandos den respuesta de manera autónoma sobre la estructura de ese contenido? ¿Cuáles son las situaciones, observaciones, intuiciones, intentos, modelos apropiados para que con un ejemplo, con un caso elemental se haga 
productivo el principio de una cosa, la estructura de un contenido y se puede aplicar y a partir de ahí ejercitar?

La didáctica aparece, entonces, como una teoría de los contenidos formativos, y la metódica como una parte complementaria, aunque esta última es relevante en la medida que la didáctica no alcanza a cubrir las problemáticas relacionadas con las formas, caminos, medios y métodos pedagógicos que son problematizados con la experiencia misma. Cabe anotar que no hay una relación de subordinación entre ambos campos, sino de interacción y complementación. Puedo señalar que, para Klafki, la didáctica, en sentido amplio, puede ser entendida como una teoría de los contenidos formativos y como ciencia del enseñar y del aprender; en sentido estricto, puede asumirse como la que designa la teoría de las tareas, categorías y contenidos formativos, de su sentido formativo. Por otra parte, la metódica se pregunta por los métodos, formas y medios de la enseñanza y aprendizaje consciente.

En la planeación didáctica se debe considerar una serie de elementos que permitirán concretar las propuestas de la Pedagogía Crítica Constructiva y de la Didáctica TeóricoFormativa; como punto inicial se debe realizar un análisis de las condiciones concretas de partida mediadas socioculturalmente correspondientes a un grupo de aprendizaje o clase, de las y los aprendices, así como el análisis de las condiciones institucionales relevantes para la enseñanza (modificables o no modificables a corto plazo) y, finalmente, de las dificultades o perturbaciones posibles o verdaderas.

Es decir, en la enseñanza de la filosofía, desde lo propuesto por Klafki, debemos partir de un contexto de fundamentación, de una estructuración temática, de la determinación de las posibilidades de acceso y presentación y de una estructuración metódica. En el contexto de fundamentación se debe considerar la importancia para el presente, para el futuro y la importancia general expresada en las metas generales del proceso de enseñanza o del centro educativo; en la estructura temática se consideran las metas sociales y parciales de aprendizaje, acompañadas de la posibilidad y probabilidad de comprobación; a continuación, en lo correspondiente a determinación de posibilidades de acceso, se debe hacer la pregunta por la accesibilidad y presentabilidad de los contenidos, por ejemplo: el uso de medios para ello; por último, en la parte de estructuración metódica se requiere organizar el proceso de enseñanza y aprendizaje, entendido como concepto variable de formas de organización y realización del aprendizaje y de las ayudas para el maestro, y también como medio y estructura interaccionales de los procesos sociales de aprendizaje.

Klafki le evita a las didácticas especiales, en este caso a la didáctica de la Filosofía, caer en la instrumentalización acrítica, y nos obliga a recordar que todo intento didáctico o metódico requiere de reflexión pedagógica para evitar su parcialización y su recorte.

\section{Bibliografía}

Blankertz, Herwig: "Didáctica". En: Speck, J., Wehle, G y otros: Conceptos fundamentales de pedagogogía. Barcelona: Herder, 1981.

KLAFKI, Wolfgang: Studien zur Bildungstheorie und Didaktik. Weinheim; Basel: Beltz, 1975. 
: "La importancia de las teorías clásicas de la educación para una concepción de la educación general hoy". En: Educación (Tubingen), vol. 36, 1985.

KoHan, Walter O. (Comp.): Teoría y práctica en filosofía con niños y jóvenes. Argentina: Noveduc, 2006.

Runge Peña, Andrés Klaus: Ensayos sobre pedagogía alemana. Bogotá: Universidad Pedagógica Nacional- Museo Pedagógico Colombiano, 2008. 